\title{
Comparison of hepatic arterial infusion chemotherapy and sorafenib in elderly patients with advanced hepatocellular carcinoma: A case series
}

\author{
TOMOYUKI NEMOTO, HIDETAKA MATSUDA, TAKUTO NOSAKA, YASUSHI SAITO, YOSHIHIKO OZAKI, \\ RYOKO HAYAMA, TATSUSHI NAITO, KAZUTO TAKAHASHI, KAZUYA OFUJI, MASAHIRO OHTANI, \\ KATSUSHI HIRAMATSU, HIROYUKI SUTO and YASUNARI NAKAMOTO
}

Division of Gastroenterology, Second Department of Internal Medicine,

Faculty of Medical Sciences, University of Fukui, Fukui 910-1193, Japan

Received May 30,2014; Accepted July 8, 2014

DOI: $10.3892 / \mathrm{mco} .2014 .371$

\begin{abstract}
Sorafenib and hepatic arterial infusion chemotherapy (HAIC) are both indicated for unresectable hepatocellular carcinoma (HCC). In this study, we compared the efficacy and safety of HAIC to that of sorafenib in elderly patients with HCC. Eligible patients included those aged $\geq 70$ years, with histologically or clinically confirmed advanced HCC. A total of 12 patients received sorafenib ( $800 \mathrm{mg}$ per day) and 8 patients received HAIC with 5 -fluorouracil $\left(300 \mathrm{mg} / \mathrm{m}^{2}\right.$ on days $1-5$ and $8-12)$ with or without cisplatin $\left(20 \mathrm{mg} / \mathrm{m}^{2}\right.$ on days 1 and 8), with interferon- $\alpha$ (3 times per week for 4 weeks). The response rate was significantly higher in patients treated with HAIC (37.5\%) compared to that in patients treated with sorafenib (no response). The median overall survival (18.6 and 11.7 months) and progression-free survival (4.0 and 5.0 months) were similar between the sorafenib and HAIC groups, respectively. In the sorafenib group, $58.3 \%$ of the patients discontinued treatment compared to none in the HAIC group. The most frequent adverse event leading to discontinuation of sorafenib was anorexia. Similar to sorafenib, HAIC appears to be a feasible treatment and may also have the advantage of an adequate safety profile for elderly patients with advanced HCC. Further study of HAIC in a larger population of elderly patients is required to assess its potential as an alternative to sorafenib for HCC.
\end{abstract}

Correspondence to: Professor Yasunari Nakamoto, Division of Gastroenterology, Second Department of Internal Medicine, Faculty of Medical Sciences, University of Fukui, 23-3 Matsuokashimoaitsuki, Fukui 910-1193, Japan

E-mail: ynakamot@u-fukui.ac.jp

Key words: hepatic arterial infusion chemotherapy, interferon, sorafenib, hepatocellular carcinoma, elderly patients, alternative treatment

\section{Introduction}

Hepatocellular carcinoma ( $\mathrm{HCC}$ ) is the most common neoplasm worldwide (1). HCC principally develops on a background of chronic liver disease, particularly cirrhosis caused by hepatitis C or hepatitis B virus infection (1). In Japan, the median age of patients with $\mathrm{HCC}$ has been increasing gradually since 1986 (2). Elderly cancer patients often present with multiple comorbidities and age-related changes in the pharmacokinetics and pharmacodynamics of anticancer drugs that may affect chemotherapeutic regimens (3). The clinical benefits of treatment of elderly patients with advanced HCC remain unclear. A previously published study demonstrated that investigations in elderly patients were less intense, that such patients were more likely to receive conservative therapy and that the median survival was worse compared to that among younger patients (4). However, the treatments for HCC have progressed significantly over the last few years and Mirici-Cappa et al (5) demonstrated that the overall applicability of radical or effective HCC treatment may not be affected by age. Moreover, Suda et al (2) suggested that the therapeutic approach to HCC should not be restricted by patient age.

Sorafenib is an oral tyrosine kinase inhibitor that targets multiple molecular pathways. In a pivotal study, sorafenib provided an overall survival (OS) advantage in patients with advanced $\mathrm{HCC}$, with the median survival increasing by $\sim 3$ months in sorafenib-treated patients, compared to those receiving placebo therapy (6). Sorafenib is the only globally approved drug for the treatment of HCC; however, it is not curative and is only indicated for Child-Pugh class A patients who have preserved hepatic function. Hepatic arterial infusion chemotherapy (HAIC) is an alternative option for advanced HCC and, based on the Japanese HCC management guidelines, it is recommended for patients with the same indications for sorafenib (7). Although HAIC is widely used in Japan, as it tends to be associated with a favorable response rate (RR) in patients with HCC, randomized controlled trials have not been conducted and there is currently no evidence of a survival benefit for HAIC. HAIC may reduce HCC stage (8) and is indicated for patients exhibiting a moderate reduction in 
hepatic reserve function (9). In patients who achieve a complete response (CR) with HAIC, a long-term survival benefit was reported $(10,11)$. The efficacy of sorafenib treatment in elderly patients with advanced HCC has been investigated in several studies (12-16); however, to the best of our knowledge, there are no available reports regarding the efficacy of HAIC in such patients and there are currently no satisfactory strategies for the management of advanced HCC as a function of age. The aim of this study was to compare the feasibility and safety of HAIC to those of sorafenib in elderly patients with advanced HCC.

\section{Patients and methods}

Patients. We retrospectively analyzed data from elderly patients with advanced unresectable HCC, who were treated at our hospital between March, 2002 and June, 2013. Eligible patients included those aged $\geq 70$ years with histologically or clinically confirmed advanced HCC. HCC was considered as unresectable in patients who presented with severe vascular invasion or multiple intrahepatic lesions (i.e., $\geq 5$ nodules), or in those with progressive disease (PD) following surgical or locoregional therapy intervention. A total of 20 eligible patients were identified.

The study protocol was approved by our Institutional Review Board and informed consent was obtained from all the patients prior to treatment.

Treatment. In the HAIC group $(\mathrm{n}=8)$, an implantable drug delivery system was used for arterial infusion of the chemotherapeutic agents. Between February, 2003 and March, 2009, HAIC consisted of 5-fluorouracil (5-FU) at a dose of $300 \mathrm{mg} / \mathrm{m}^{2} /$ day for 5 days during the 1 st and 2nd weeks, combined with intramuscular or subcutaneous administration of interferon- $\alpha 3$ times per week for 4 weeks. Interferon- $\alpha$ dosing consisted of either natural interferon- $\alpha$, 5 million units; recombinant interferon- $\alpha, 12$ million units; or interferon- $\alpha$ 2b, 3 million units. From April, 2009 onwards, HAIC was performed with 5-FU plus cisplatin (CDDP) at a dose of $20 \mathrm{mg} / \mathrm{m}^{2} /$ day on days 1 and 8 , combined with intramuscular interferon- $\alpha$ administration, as described above (17). The treatment cycle was repeated until disease progression or unacceptable drug toxicity.

In the sorafenib group $(n=12)$, a limited number of patients received sorafenib $200-600 \mathrm{mg} /$ day as an initial dose. In the absence of adverse events (AEs), the dose of sorafenib was increased to $400 \mathrm{mg}$ twice daily. Treatment was discontinued on the same basis as in the HAIC group. However, if the performance status and liver function of patients with PD was preserved, sorafenib was continued until the occurrence of severe AEs in order to prevent rapid tumor growth associated with treatment cessation.

Response assessment. Tumor response was determined using dynamic computed tomography or magnetic resonance imaging, according to Response Evaluation Criteria in Solid Tumors, version 1.1. RR was defined as the combined percentages of patients experiencing a CR and those with a partial response (PR). Tumor control rate (TCR) was defined as the combined percentages of patients experiencing $\mathrm{CR}, \mathrm{PR}$ and
Table I. Clinical characteristics of patients treated with sorafenib and hepatic arterial infusion chemotherapy (HAIC).

\begin{tabular}{|c|c|c|c|}
\hline Variables & $\begin{array}{l}\text { Sorafenib } \\
(\mathrm{n}=12)\end{array}$ & $\begin{array}{l}\text { HAIC } \\
(\mathrm{n}=8)\end{array}$ & P-value \\
\hline Age (years) & $80.2 \pm 5.4$ & $74.9 \pm 3.4$ & $0.039^{\mathrm{a}}$ \\
\hline Gender $(\mathrm{M} / \mathrm{F})$ & $6 / 6$ & $6 / 2$ & $\mathrm{NS}^{\mathrm{b}}$ \\
\hline $\begin{array}{l}\text { White cell count } \\
\left(\mathrm{x} 10^{2} / \mu \mathrm{l}\right)\end{array}$ & $48.0 \pm 13.2$ & $59.4 \pm 27.4$ & $\mathrm{NS}^{\mathrm{a}}$ \\
\hline $\begin{array}{l}\text { Lymphocyte count } \\
\left(\mathrm{x} 10^{2} / \mu 1\right)\end{array}$ & $14.6 \pm 8.5$ & $14.9 \pm 6.2$ & $\mathrm{NS}^{\mathrm{a}}$ \\
\hline $\begin{array}{l}\text { Platelet count } \\
\left(\mathrm{x} 10^{4} / \mu \mathrm{l}\right)\end{array}$ & $16.7 \pm 6.1$ & $14.1 \pm 6.7$ & $\mathrm{NS}^{\mathrm{a}}$ \\
\hline PT-INR & $1.10 \pm 34.6$ & $1.16 \pm 0.19$ & $\mathrm{NS}^{\mathrm{a}}$ \\
\hline ALT (IU/l) & $35.5 \pm 0.12$ & $41.9 \pm 0.19$ & $\mathrm{NS}^{\mathrm{a}}$ \\
\hline $\begin{array}{l}\text { Total bilirubin } \\
(\mathrm{mg} / \mathrm{dl})\end{array}$ & $0.67 \pm 0.40$ & $0.76 \pm 0.27$ & $\mathrm{NS}^{\mathrm{a}}$ \\
\hline Albumin (g/dl) & $3.5 \pm 0.4$ & $3.3 \pm 0.8$ & $\mathrm{NS}^{\mathrm{a}}$ \\
\hline $\begin{array}{l}\text { Cirrhosis } \\
\text { (Child-Pugh A/B/C) }\end{array}$ & $10 / 2 / 0$ & $4 / 4 / 0$ & $\mathrm{NS}^{\mathrm{b}}$ \\
\hline $\begin{array}{l}\text { TNM stage } \\
\text { (I/II/III/IV-A/IV-B) }\end{array}$ & $0 / 2 / 3 / 2 / 5$ & $0 / 2 / 3 / 2 / 1$ & $\mathrm{NS}^{\mathrm{c}}$ \\
\hline Largest tumor (mm) & $42.3 \pm 21.2$ & $49.7 \pm 28.2$ & $\mathrm{NS}^{\mathrm{a}}$ \\
\hline AFP & $2,027 \pm 5,219$ & $279 \pm 418$ & $\mathrm{NS}^{\mathrm{a}}$ \\
\hline
\end{tabular}

Results are expressed as means \pm standard deviation. ${ }^{\mathrm{a}}$ Mann-Whitney $\mathrm{U}$ test. ${ }^{b}$ Fisher's exact test. ${ }^{\mathrm{c}} \mathrm{Chi}$-square test. M, male; F, female; NS, non-significant; PT-INR, prothrombin time-international normalized ratio; ALT, alanine aminotransferase; Child-Pugh, Child-Pugh classification; TNM, tumor-node-metastasis; AFP, $\alpha$-fetoprotein

Table II. Comparison of best response between sorafenib and hepatic arterial infusion chemotherapy (HAIC).

\begin{tabular}{llcc}
\hline Response & $\begin{array}{c}\text { Sorafenib } \\
(\mathrm{n}=12)\end{array}$ & $\begin{array}{c}\text { HAIC } \\
(\mathrm{n}=8)\end{array}$ & P-value \\
\hline CR & $0(0.0)$ & $1(12.5)$ & $\mathrm{NS}$ \\
PR & $0(0.0)$ & $2(25.0)$ & $\mathrm{NS}$ \\
SD & $6(50.0)$ & $4(50.0)$ & $\mathrm{NS}$ \\
PD & $6(50.0)$ & $1(12.5)$ & $\mathrm{NS}$ \\
RR (CR+PR) & $0(0.0)$ & $3(37.5)$ & $0.049^{\mathrm{a}}$ \\
TCR (CR+PR+SD) & $6(50.0)$ & $7(87.5)$ & $\mathrm{NS}$ \\
\hline
\end{tabular}

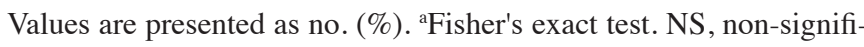
cant; CR, complete response; $\mathrm{PR}$, partial response; $\mathrm{SD}$, stable disease; $\mathrm{PD}$, progressive disease; $\mathrm{RR}$, response rate; TCR, tumor control rate.

stable disease (SD). HAIC was evaluated every 6 or 8 weeks and sorafenib treatment was evaluated every 4 or 12 weeks. OS was calculated from the date of treatment initiation to the date of the last follow-up or death. Progression-free survival (PFS) was calculated from the date of treatment initiation to the date 
A

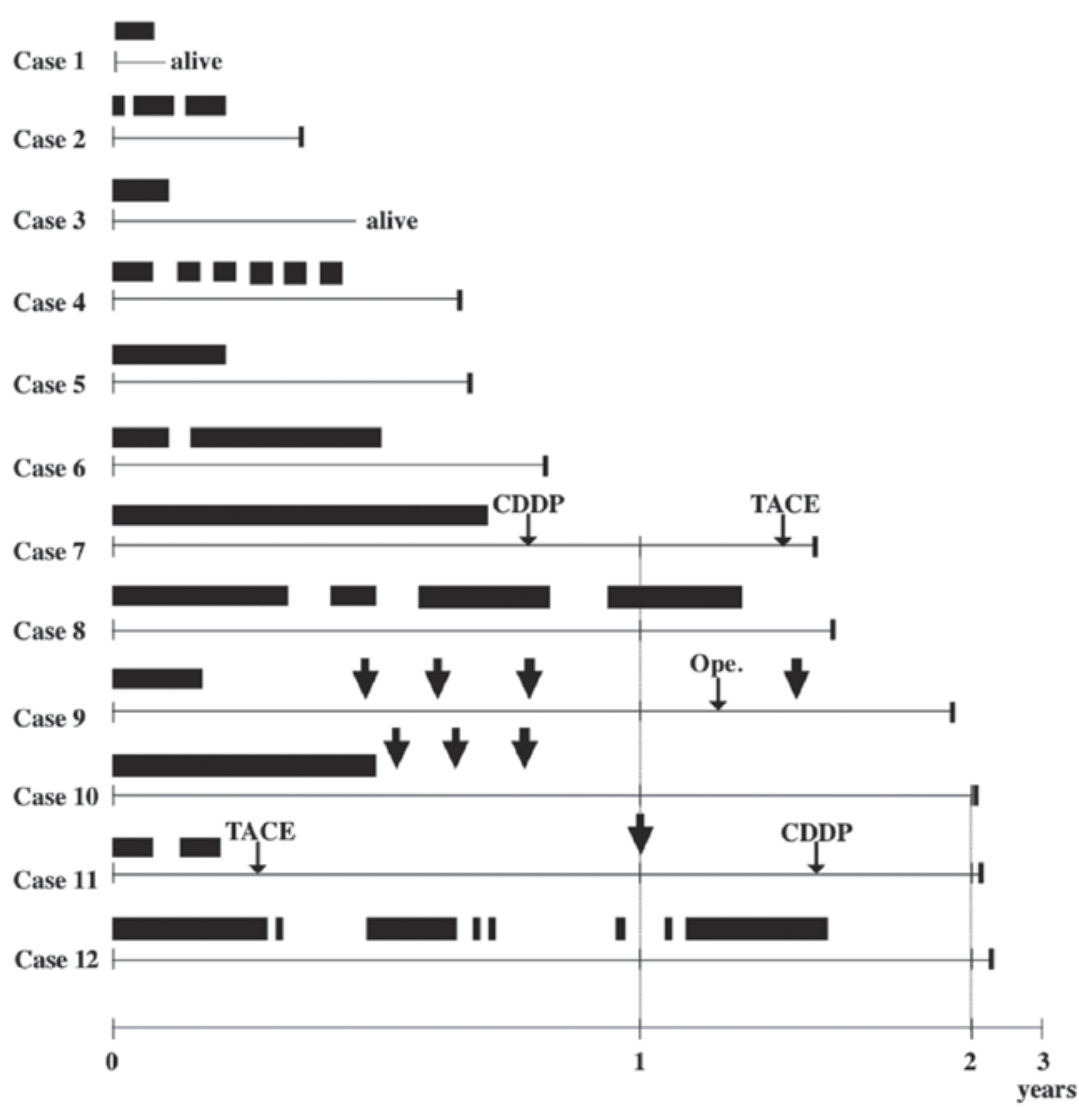

B

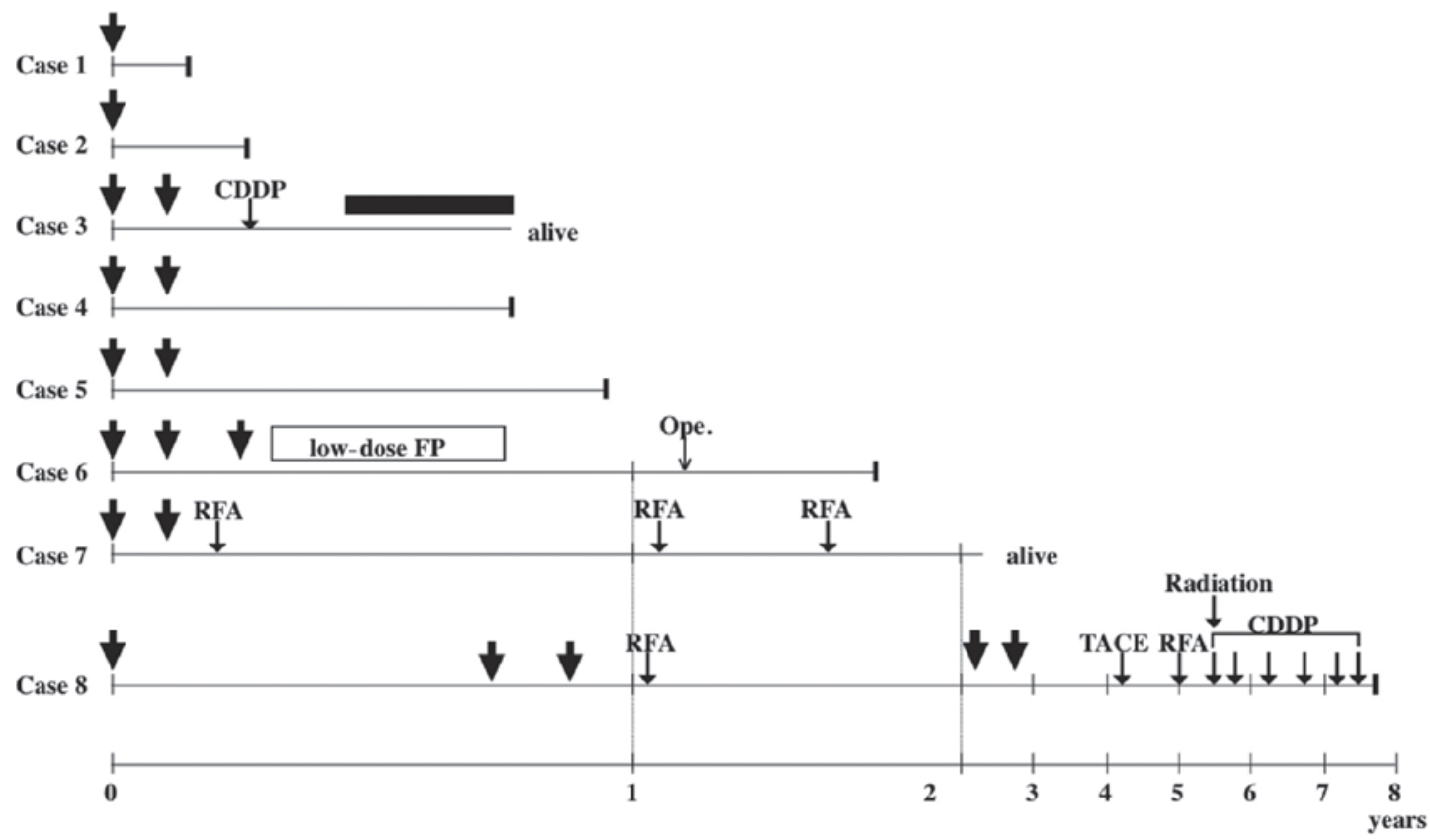

Figure 1. Clinical course of (A) the sorafenib and (B) hepatic arterial infusion chemotherapy (HAIC) groups. The best clinical responses were complete response in 1 patient (case 8) in the HAIC group, partial response in 2 patients (cases 6 and 7) in the HAIC group, stable disease in 10 patients (cases 1, 6, 9, 10, 11 and 12 in the sorafenib group and cases 2,3,4 and 5 in the HAIC group) and progressive disease in 7 patients (cases 2, 3, 4, 5, 7 and 8 in the sorafenib group and case 1 in the HAIC group). Although patients 1 and 3 in the sorafenib group and patients 3 and 7 in the HAIC group remained alive, other patients succumbed to the disease at the indicated time points. Closed bars, sorafenib administration. Arrows, HAIC. CDDP, cisplatin infusion; TACE, transcatheter arterial chemoembolization; Ope., operation; low-dose FP, continuous 5-fluorouracil and low-dose cisplatin infusion; RFA, radiofrequency ablation.

of the last follow-up or PD. Drug-related AEs were evaluated according to the Common Toxicity Criteria for Adverse Events, version 4.0 (Japan Clinical Oncology Group/Japan Society of Clinical Oncology edition).
Additional therapy. Of the 20 patients, 8 received additional treatment, including surgery, radiofrequency ablation (RFA), transcatheter arterial chemoembolization (TACE), HAIC using 5-FU and low-dose CDDP without interferon- $\alpha$ 
administration (low-dose FP), arterial CDDP infusion and irradiation therapy.

Statistical analyses. The results are expressed as means \pm standard deviation. The differences between the two groups were examined for statistical significance using the Mann-Whitney U test, the Fisher's exact test and the Chi-square test. The survival curves for OS and PFS were analyzed using the Kaplan-Meier method and the differences were evaluated using a log-rank test. The $95 \%$ confidence intervals (CIs) of median OS and median PFS were calculated. $\mathrm{P}<0.05$ was considered to indicate a statistically significant difference.

\section{Results}

Patient characteristics. The baseline patient clinical characteristics are summarized in Table I. The mean age of the sorafenib group was significantly higher compared to that of the HAIC group $(\mathrm{P}=0.039)$. There were no significant differences by blood cell counts, blood coagulation tests, biochemical tests, or Child-Pugh classifiction. In addition, a comparison of tumor-related background factors between the two groups did not reveal any significant differences in TNM stage, main tumor diameter, or serum $\alpha$-fetoprotein levels.

Clinical response. The mean daily dose and duration of sorafenib treatment were $544 \mathrm{mg}$ and 5.3 months, respectively. The mean number of treatment cycles in the HAIC group was 1.8 ( 2.2 months). The treatment responses are summarized in Table II. The RR was significantly different between the two groups, as patients in the sorafenib group failed to respond to treatment $(\mathrm{P}=0.049)$. However, there was no significant difference in TCR between the two groups. Two patients in the HAIC group achieved a sustained CR after receiving additional RFA: one initially achieved a CR in response to HAIC and the other initially demonstrated a PR in response to HAIC.

Clinical course and additional therapy. Fig. 1 shows the clinical course of patients who were treated with sorafenib (Fig. 1A) or HAIC (Fig. 1B). In the sorafenib group, treatment was discontinued in 11 patients; for 7 patients (patients 2, 3, 4, 5, 7, 9 and 11), this was due to drug-related AEs, whereas the remaining patients (patients 6, 8, 10 and 11) developed PD. In the HAIC group, none of the patients discontinued 5-FU and CDDP infusion, but interferon- $\alpha$ administration was discontinued in 1 patient (patient 1). Four patients in each group (patients 7, 9, 10 and 11, Fig. 1A; and patients 3, 6, 7 and 8, Fig. 1B) received various additional therapies, including arterial CDDP infusion (patients 7 and 11, Fig. 1A; and patients 3 and 8, Fig. 1B); operation (patient 9, Fig. 1A; and patient 6, Fig. 1B); TACE (patients 7 and 11, Fig. 1A; and patient 8, Fig. 1B); low-dose FP (patient 6, Fig. 1B); RFA (patients 7 and 8, Fig. 1B); and radiation therapy (patient 8, Fig. 1B). Patients 9 and 10 (Fig. 1A) underwent HAIC immediately after sorafenib failure, whereas patient 3 (Fig. 1B) received sorafenib immediately after HAIC failure. Overall, no patients in the sorafenib group demonstrated a curative response following these treatments, whereas for 3 patients in the HAIC group, the additional treatment was significantly curative $(\mathrm{P}=0.049)$.
A
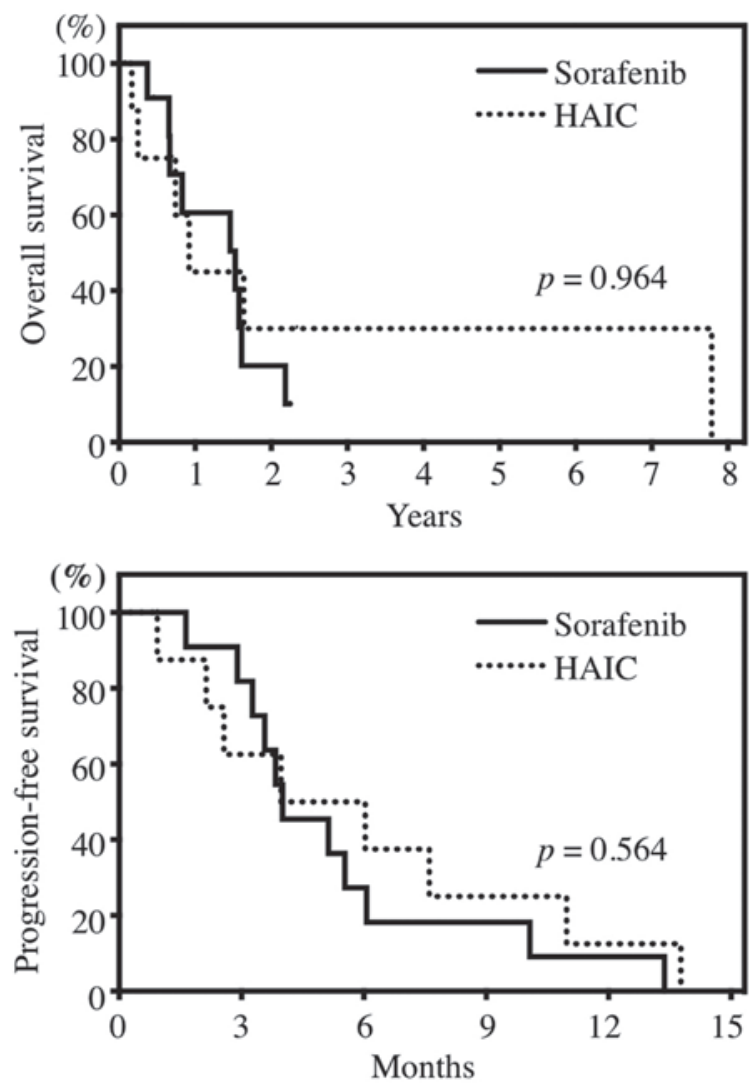

Figure 2. Kaplan-Meier analysis of (A) overall survival and (B) progression-free survival according to sorafenib and hepatic arterial infusion chemotherapy (HAIC). The P-value was calculated using the log-rank test.

Survival. The median OS of the total patient population was 17.8 months (0.93-94.7 months). The median OS was 18.6 months (95\% CI: 13.8-23.4) and 11.7 months (95\% CI: 0-31.5) in the sorafenib and HAIC groups, respectively (Fig. 2A). The median PFS was 4.0 months (95\% CI: 2.1-5.9) and 5.0 months (95\% CI: 2.6-7.4) in the sorafenib and HAIC groups, respectively (Fig. 2B). The median OS and PFS were not significantly different between the two groups $(\mathrm{P}=0.964$ and 0.562 , respectively).

Safety. The major AEs are listed in Table III. A total of 7 patients $(58.3 \%)$ in the sorafenib group discontinued treatment due to grade 3 AEs [4 patients, anorexia; and 1 patient each with hand-foot (HF) syndrome, ascites and hepatic encephalopathy], whereas no patients demonstrated intolerance to HAIC. The discontinuation rate in the sorafenib group was significantly higher compared to that in the HAIC group $(\mathrm{P}=0.015)$. Among sorafenib-treated patients, the most frequent AEs were mild in severity (grade 1/2) and included HF syndrome, anorexia, hypoalbuminemia and diarrhea. Grade 3 AEs included HF syndrome, anorexia and hypertension. One Child-Pugh class A patient developed hepatic failure (hepatic encephalopathy) and sorafenib was discontinued. There were no grade 4 AEs. Among HAIC group patients, the most frequent AEs were mostly mild in severity (grade 1/2) and included decreased platelet count, anemia, fever, malaise, anorexia, hypoalbuminemia, decreased white blood cell count and decreased neutrophil count. In total, 6 hematological 
Table III. Adverse events.

\begin{tabular}{|c|c|c|c|c|c|c|c|c|c|c|c|c|}
\hline \multirow[b]{2}{*}{ Adverse events } & \multicolumn{6}{|c|}{$\begin{array}{c}\text { Sorafenib }(\mathrm{n}=12) \\
\text { Grade (CTCAE v4.0) }\end{array}$} & \multicolumn{6}{|c|}{$\begin{array}{c}\text { HAIC }(\mathrm{n}=8) \\
\text { Grade (CTCAE v4.0) }\end{array}$} \\
\hline & 1 & 2 & 3 & 4 & Any & $3-4$ & 1 & 2 & 3 & 4 & Any & $3-4$ \\
\hline Anemia & 4 & 1 & & & $5(41.7)$ & 0 & 2 & 5 & & & $7(87.5)$ & 0 \\
\hline Decreased WBC & & 1 & & & $1(8.3)^{\mathrm{a}}$ & 0 & 2 & 3 & 1 & & $6(75.0)^{\mathrm{a}}$ & $1(12.5)$ \\
\hline Decreased neutrophil count & & 1 & & & $1(8.3)^{\mathrm{a}}$ & 0 & 3 & 2 & 1 & & $6(75.0)^{\mathrm{a}}$ & $1(12.5)$ \\
\hline Decreasedlatelet count & 4 & 3 & 1 & & $8(66.7)$ & $1(8.3)$ & 3 & & 3 & 1 & $7(87.5)$ & $4(50.0)$ \\
\hline Malaise & 5 & 2 & & & $7(58.3)$ & 0 & 4 & 3 & & & $7(87.5)$ & 0 \\
\hline Fever & & & & & $0^{\mathrm{b}}$ & 0 & 6 & 1 & & & $7(87.5)^{b}$ & 0 \\
\hline Anorexia & 2 & 4 & 3 & & $9(75.0)$ & $3(25.0)$ & 3 & 4 & & & $7(87.5)$ & 0 \\
\hline Nausea & 1 & & & & $1(8.3)$ & 0 & 2 & & & & $2(25.0)$ & 0 \\
\hline Vomiting & & & & & 0 & 0 & & & & & 0 & 0 \\
\hline Diarrhea & 2 & 7 & & & $9(75.0)$ & 0 & 2 & & & & $2(25.0)$ & 0 \\
\hline Mucositis & 1 & 2 & & & $3(25.0)$ & 0 & & 1 & & & $1(12.5)$ & 0 \\
\hline Hand-foot syndrome & 4 & 1 & 4 & & $9(75.0)^{\mathrm{a}}$ & $4(33.3)$ & & & & & $0^{\mathrm{a}}$ & 0 \\
\hline Hepatic encephalopathy & & & 1 & & $1(8.3)$ & $1(8.3)$ & & & & & 0 & 0 \\
\hline Ascites & & 3 & & & $3(25.0)$ & 0 & & & & & 0 & 0 \\
\hline Bleeding & & & & & 0 & 0 & & & & & 0 & 0 \\
\hline Cardiological & & & & & 0 & 0 & & & & & 0 & 0 \\
\hline Hypertension & 2 & 3 & 3 & & $8(66.7)^{\mathrm{a}}$ & $3(25.0)$ & & & & & $0^{\mathrm{a}}$ & 0 \\
\hline Pancreatitis & & & & & 0 & 0 & & & & & 0 & 0 \\
\hline Infection & & 1 & & & $1(8.3)$ & 0 & & 2 & & & $2(25.0)$ & 0 \\
\hline Hyperbilirubinemia & 2 & 1 & & & $3(25.0)$ & 0 & 2 & & & & $2(25.0)$ & 0 \\
\hline Hypoalbuminemia & 2 & 8 & & & $10(83.3)$ & 0 & 1 & 5 & & & $6(75.0)$ & 0 \\
\hline Increased AST & 6 & & & & $6(50.0)$ & 0 & 2 & & & & $2(25.0)$ & 0 \\
\hline Increased ALT & 3 & & & & $3(25.0)$ & 0 & 1 & & & & $1(12.5)$ & 0 \\
\hline Increased creatinine & 1 & & & & $1(8.3)$ & 0 & 1 & 2 & & & $3(37.5)$ & 0 \\
\hline Increased serum amylase & 3 & 2 & & & $5(41.7)$ & 0 & & & & & 0 & 0 \\
\hline
\end{tabular}

The values represent number of events and the parenthetical data represent percentage values. ${ }^{\mathrm{a}} \mathrm{P}<0.01$; ${ }^{\mathrm{b}} \mathrm{P}<0.001$ (Fisher's exact test). CTCAE v4.0, Common Terminology Criteria for Adverse Events, version 4.0; WBC, white blood cell; AST, aspartate aminotransferase; ALT, alanine aminotransferase.

AEs of grade $3 / 4$ were recorded in 4 patients. In the HAIC group, 1 patient $(12.5 \%)$ experienced catheter occlusion as a catheter-related complication. In addition, 5 patients in the sorafenib group changed Child-Pugh class from A to B, whereas none of the patients in the HAIC group changed Child-Pugh class. These changes were mostly caused by the development of hypoalbuminemia in sorafenib-treated patients; there was no significant change in the prothrombin time-international normalized ratio (PT-INR).

\section{Discussion}

In the present study, we demonstrated the feasibility and safety of HAIC in elderly patients with advanced HCC. Several previous studies demonstrated the efficacy and safety of sorafenib in elderly patients $(12,14-16)$; however, to the best of our knowledge, there are no studies performing a comparison of efficacy and safety between sorafenib and HAIC in elderly patients with HCC. It should be noted that the definition of 'elderly' may be controversial. We selected the cut-off age of
70 years, as the majority of age-related changes occur after this age (3). There are some studies available comparing sorafenib and HAIC for the treatment of HCC, but they were not performed in elderly patients $(18,19)$.

In the present study, the RR of the HAIC group was significantly higher compared to that of the sorafenib group, but the TCR was similar between the two groups. Our findings were concurrent with those of previous studies of interferon- $\alpha$-containing HAIC that demonstrated a RR of $24.6-73.0 \%(11,17,20-24)$, indicating that interferon- $\alpha$-containing HAIC is a feasible treatment for elderly patients with advanced HCC.

An important finding of the present study is that, in the HAIC group, over a third of the patients achieved a CR or PR and, among these patients, 3 achieved long-term survival with additional curative therapy. This observation has important implications in understanding the indications for HAIC in elderly patients. There were no significant differences in median OS and PFS between the two groups. The median PFS with sorafenib was similar to that reported by previous 
investigations in elderly patients, but the median OS was longer (12,14-16). The reasons underlying the prolongation of OS in the sorafenib group in the present study are unknown, but one possibility is that the sorafenib group included 2 patients who received HAIC immediately after disease progression, which may skew the data. Two patients in the HAIC group achieved a CR after additional RFA. Other studies have demonstrated that a CR may improve long-term survival, although this was demonstrated in elderly patients $(10,11)$.

The rate of treatment discontinuation due to severe AEs was significantly higher in the sorafenib group compared to that in the HAIC group. Multiple AEs have been associated with 5-FU, CDDP and interferon- $\alpha$ therapy; however, life-threatening AEs rarely occur, even in patients with liver cirrhosis $(11,17,20,23)$. In this study, AEs in HAIC-treated patients were more severe than previously reported $(11,17,20,23)$, particularly thrombocytopenia, although none resulted in treatment discontinuation or required any additional management. The evaluation of AEs in this patient population may be challenging, as the majority of the patients already presented with pancytopenia due to underlying liver cirrhosis. However, a high AE-induced discontinuation rate was apparent among sorafenib-treated patients, mostly as a result of anorexia or hypoalbuminemia, which may lead to ascites. In the present study, patients with a mean age of 80.2 years comprised $75 \%$ of all the grades of anorexia. This is concordant with the observations of Morimoto et al (13), who indicated that the incidence of anorexia was significantly higher among patients aged $\geq 75$ years. Our results and those of Morimoto et al (13) differ from the results of the SHARP and Asia-Pacific trials (6,25); however, in those studies, the age and incidence of all-grade anorexia was 64.9 years (mean) and 51 years (median) and 14 and $12.8 \%$, respectively $(6,25)$. The results of those studies and our present results suggest that elderly patients are more prone to sorafenib-induced anorexia. In addition, Montella et al (15) suggested that the changes reported in Child-Pugh scores, as a result of changes in hypoalbuminemia and PT-INR, appeared to be associated with liver function and worsening of cirrhosis, rather than to the drugs administered. However, in the elderly patients in this study, the PT-INR did not change, suggesting preserved hepatic protein synthesis, indicating that hypoalbuminemia may be associated with the anorexia, rather than liver dysfunction. Accordingly, the results of the present study suggest that hypoalbuminemia is an important AE in elderly patients. In summary, HAIC may be a safer option compared to sorafenib for the treatment of elderly patients with HCC.

There were several limitations in the interpretation of the data presented in this study. First, the retrospective design and limited number of patients enrolled may give rise to selection bias. The mean age of the sorafenib group was higher compared to that of the HAIC group, which may explain why the incidence of AEs was higher in the sorafenib group. Moreover, according to the initial response to treatment, additional therapies were performed without limitation, which may affect OS. All the patients in the sorafenib group who received additional therapies developed PD or severe AEs, while some of the patients in the HAIC group who received additional therapies achieved a CR or PR. However, in part, the present study provided significant information regarding the management of HCC in elderly patients.
In conclusion, HAIC appears to be a feasible and safe treatment option for elderly patients with advanced HCC. However, further study of HAIC in a larger population of elderly patients is required to assess its potential as an alternative option for HCC management.

\section{Acknowledgements}

This study was supported, in part, by a grant from the Clinical Trial and Advanced Medical Center of University of Fukui.

\section{References}

1. El-Serag HB: Epidemiology of viral hepatitis and hepatocellular carcinoma. Gastroenterology 142: 1264-1273, 2012.

2. Suda T, Nagashima A, Takahashi S, et al: Active treatments are a rational approach for hepatocellular carcinoma in elderly patients. World J Gastroenterol 19: 3831-3840, 2013.

3. Balducci L: Geriatric oncology: challenges for the new century. Eur J Cancer 36: 1741-1754, 2000.

4. Collier JD, Curless R, Bassendine MF and James OF: Clinical features and prognosis of hepatocellular carcinoma in Britain in relation to age. Age Ageing 23: 22-27, 1994

5. Mirici-Cappa F, Gramenzi A, Santi V, et al: Treatments for hepatocellular carcinoma in elderly patients are as effective as in younger patients: a 20-year multicentre experience. Gut 59: 387-396, 2010.

6. Llovet JM, Ricci S, Mazzaferro V, et al: Sorafenib in advanced hepatocellular carcinoma. N Engl J Med 359: 378-390, 2008.

7. Kudo M, Izumi N, Kokudo N, et al; HCC Expert Panel of Japan Society of Hepatology: Management of hepatocellular carcinoma in Japan: Consensus-Based Clinical Practice Guidelines proposed by the Japan Society of Hepatology (JSH) 2010 updated version. Dig Dis 29: 339-364, 2011.

8. Meric F, Patt YZ, Curley SA, et al: Surgery after downstaging of unresectable hepatic tumors with intra-arterial chemotherapy. Ann Surg Oncol 7: 490-495, 2000.

9. Miyaki D, Aikata H, Honda Y, et al: Hepatic arterial infusion chemotherapy for advanced hepatocellular carcinoma according to Child-Pugh classification. J Gastroenterol Hepatol 27: 1850-1857, 2012.

10. Ando E, Tanaka M, Yamashita F, et al: Hepatic arterial infusion chemotherapy for advanced hepatocellular carcinoma with portal vein tumor thrombosis: analysis of 48 cases. Cancer 95: 588-595, 2002.

11. Obi S, Yoshida H, Toune R, et al: Combination therapy of intraarterial 5-fluorouracil and systemic interferon-alpha for advanced hepatocellular carcinoma with portal venous invasion. Cancer 106: 1990-1997, 2006.

12. Wong H, Tang YF, Yao TJ, et al: The outcomes and safety of single-agent sorafenib in the treatment of elderly patients with advanced hepatocellular carcinoma (HCC). Oncologist 16: 1721-1728, 2011.

13. Morimoto M, Numata $\mathrm{K}$, Kondo M, et al: Higher discontinuation and lower survival rates are likely in elderly Japanese patients with advanced hepatocellular carcinoma receiving sorafenib. Hepatol Res 41: 296-302, 2011.

14. Di Costanzo GG, Tortora R, De Luca M, et al: Impact of age on toxicity and efficacy of sorafenib-targeted therapy in cirrhotic patients with hepatocellular carcinoma. Med Oncol 30: 446, 2013.

15. Montella L, Addeo R, Cennamo G, et al: Sorafenib in elderly patients with advanced hepatocellular carcinoma: a case series. Oncology 84: 265-272, 2013.

16. Jo M, Yasui K, Kirishima T, et al: Efficacy and safety of sorafenib in very elderly patients aged 80 years and older with advanced hepatocellular carcinoma. Hepatol Res: Feb 14, 2014 (Epub ahead of print).

17. Yamashita T, Arai K, Sunagozaka H, et al: Randomized, phase II study comparing interferon combined with hepatic arterial infusion of fluorouracil plus cisplatin and fluorouracil alone in patients with advanced hepatocellular carcinoma. Oncology 81 : 281-290, 2011.

18. Hiramine Y, Uto H, Imamura Y, et al: Sorafenib and hepatic arterial infusion chemotherapy for unresectable advanced hepatocellular carcinoma: A comparative study. Exp Ther Med 2: 433-441, 2011. 
19. Jeong SW, Jang JY, Lee JE, et al: The efficacy of hepatic arterial infusion chemotherapy as an alternative to sorafenib in advanced hepatocellular carcinoma. Asia Pac J Clin Oncol 8: 164-171, 2012.

20. Kasai K, Ushio A, Kasai Y, et al: Therapeutic efficacy of combination therapy with intra-arterial 5-fluorouracil and systemic pegylated interferon alpha- $2 b$ for advanced hepatocellular carcinoma with portal venous invasion. Cancer 118: 3302-3310, 2012.

21. Enjoji M, Morizono S, Kotoh K, et al: Re-evaluation of antitumor effects of combination chemotherapy with interferon-alpha and 5-fluorouracil for advanced hepatocellular carcinoma. World J Gastroenterol 11: 5685-5687, 2005.

22. Ota H, Nagano H, Sakon M, et al: Treatment of hepatocellular carcinoma with major portal vein thrombosis by combined therapy with subcutaneous interferon-alpha and intra-arterial 5-fluorouracil; role of type 1 interferon receptor expression. Br J Cancer 93: 557-564, 2005.
23. Nagano H, Wada H, Kobayashi S, et al: Long-term outcome of combined interferon-alpha and 5-fluorouracil treatment for advanced hepatocellular carcinoma with major portal vein thrombosis. Oncology 80: 63-69, 2011.

24. Uka K, Aikata H, Takaki S, et al: Pretreatment predictor of response, time to progression, and survival to intraarterial 5 -fluorouracil/interferon combination therapy in patients with advanced hepatocellular carcinoma. J Gastroenterol 42: 845-853, 2007.

25. Cheng AL, Kang YK, Chen Z, et al: Efficacy and safety of sorafenib in patients in the Asia-Pacific region with advanced hepatocellular carcinoma: a phase III randomised, double-blind, placebo-controlled trial. Lancet Oncol 10: 25-34, 2009. 